\title{
3D numerical analysis of anti-slide pile resistance effect and coupling effect of soil-pile interaction
}

\author{
Baoqin LIAN ${ }^{1,}$,, Xingang WANG ${ }^{2, b}$, and Hui ZHONG ${ }^{1, c}$ \\ ${ }^{1}$ Department Department of Building Engineering, Guangzhou City Construction College, \\ Guangdong, Guangzhou 510925, China; ${ }^{2}$ State Key Laboratory of Continental Dynamics, \\ Department of Geology, Northwest University, Xi'an 710069, China, \\ a328602223@qq.com, ’wangxingang4202@163.com, ‘914108739@qq.com
}

\begin{abstract}
Keywords: anti-slide pile; 3D numerical analysis; pile-soil interface; soil-pile interaction
\end{abstract} Abstract. The phenomenon of the soil arch effect of anti-slide pile be discussed. Anti-slide pile resistance effect and soil-pile interaction being analyzed by three-dimensional numerical software FLAC3D With engineering examples as the background to establish numerical model, and considering the pile soil contact surface element properties. Demonstrates the reliability of numerical model by compared to the displacement of sliding zone area monitoring and scene of the field observation data analysis anti-slide pile resistance slide effect. After anti-slide pile construction, displacement and shear stress nephogram mutagenesis as oblique type of "L" shape cloud, anti-slide pile resistance slip effect is obvious. Can used the effect of soil-pile interaction effective prevention and control of landslides that was formed by anti-slide pile and soil formation, to optimize the design of the anti-slide pile when the anti-slide pile design, provides a feasible analysis method for the study of landslide prevention and control effect that has the same engineering geological conditions.

\section{Introduction}

Landslide is a common geological disasters is more outstanding in the three gorges reservoir's areas, heavy rains and reservoir water level and other factors contributed to this disaster occurs, often cause serious losses. slide-resistant pile is an effective resistance sliding structure that pile body area is large and using the interaction of pile, sliding body and sliding bed to resistance sliping ${ }^{[1]}$. When the sliding body after pile act on the pile, soil arch effect was formed because of the differences physical properties between two kinds of medium pile and soil led to the "tight" wedge effect between the soil particles ${ }^{[2]}$. Can used the effect of soil-pile interaction effective prevention and control of landslides that was formed by anti-slide pile and soil formation. The thrust after the landslide out-of-level, in the spatial distribution, coupling analysis of piles-soil in three dimensional space that Considering the interaction among piles, sliding body and sliding bed was rare in the literature ${ }^{[3-17]}$, this paper analysis of the three-dimensional space's anti-slide pile resistance effect and coupling effect of soil-pile interaction by uses the three-dimensional numerical analysis software FLAC3D.

\section{Engineering example and 3D numerical model}

Three-dimensional numerical model take majiagou 1\# landslide that in three gorges reservoir area for example, 1\# landslide is located in the left bank of the Yangtze river, about $2.1 \mathrm{~km}$ away from the Yangtze river estuary, its leading edge is below the $135 \mathrm{~m}$ water level, leading edge forming a steep slope due to river erosion, is in unstable condition, Has to governance the anti-slide measures(fig 1).

Selected the central axis section in 1\# landslide for numerical calculation model. consider effect between pile and soil, pile and pile, so selection four piles that in the central of landslide and the construction has been finished to analyze the anti-slide pile resistance effect and coupling effect of soil-pile interaction. Pile length is $18 \mathrm{~m}$ pile spacing section is $2 \mathrm{~m} \times 3 \mathrm{~m} \times 5 \mathrm{~m}$ (fig 2 ). The model using ansys software pretreatment, local fine mesh in the study area of pile and soil and slippery body sliding zone location, after that import the model to FLAC3D software, the model totals has 117544 units and 118917 nodes. In the simulation of the actual situation of pile - soil interaction should be considered 
with slip between pile and soil contact, therefore, set the pile soil contact surface between the two (fig 3). Pile-soil contact surface parameters is a key, Potyondy and Acer's research shows that the value of the anti-slide pile and soil friction angle between soil's $0.6 \sim 0.7$ times ,cohesive force value is adjacent soil's $0.7 \sim 0.8$ times is more appropriate. The normal stiffness and shear stiffness take "the hard" equivalent stiffness of the adjacent area around 10 times: Kn and Ks can use the following formula ${ }^{[18]}$ :

$$
K_{\mathrm{n}}=K_{\mathrm{s}}=10 \max \left[(K+4 G / 3) / \Delta z_{\text {min }}\right]
$$

Where: $K_{n}$ and $K_{s}$ is the normal stiffness and shear stiffness(MPa), $\Delta z_{\min }$ is the minimal size in the connection area on contact surface normal, dimensionless. Obtained the pile soil contact surface parameters (table 1, where $c$ is friction angle $\varphi$ is cohesive force) :

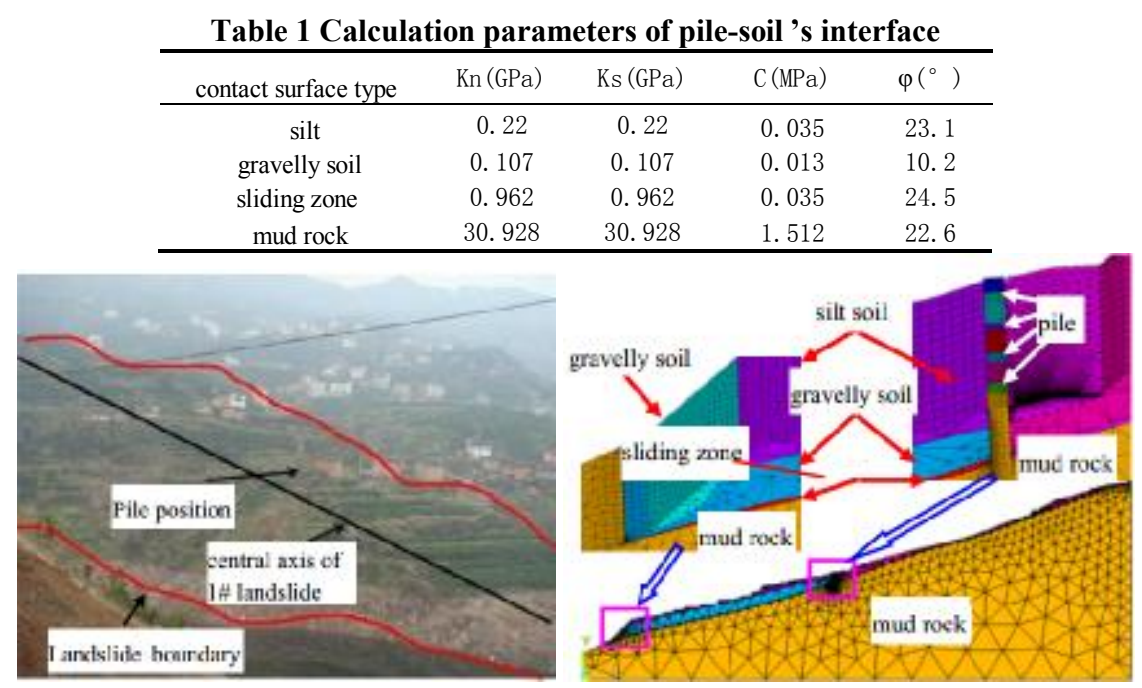

Fig.1 Sketch of Majiagou No. I landslide Fig.2 Three-dimensional model of anti-slide pile

The calculating model's bottom surface, left and right sides, pre and post side be fixed, the top surface be freeed. The result of calculation's accuracy is related to the model's parameters of each medium, rock and earth mass be used for the mohr coulomb model, anti-slide pile serve as the elastic model, the parameters obtained by laboratory test.

\section{Three-dimensional numerical analysis}

Set up a series of monitoring stations in the sliding surface location to calculation and analysis, useing 3D numerical model to demonstrates the reliability, contrast the Landslide model that not using anti-slide pile or using anti-slide pile. The results according to the calculation are as follows:

\section{Horizontal displacement analysis}
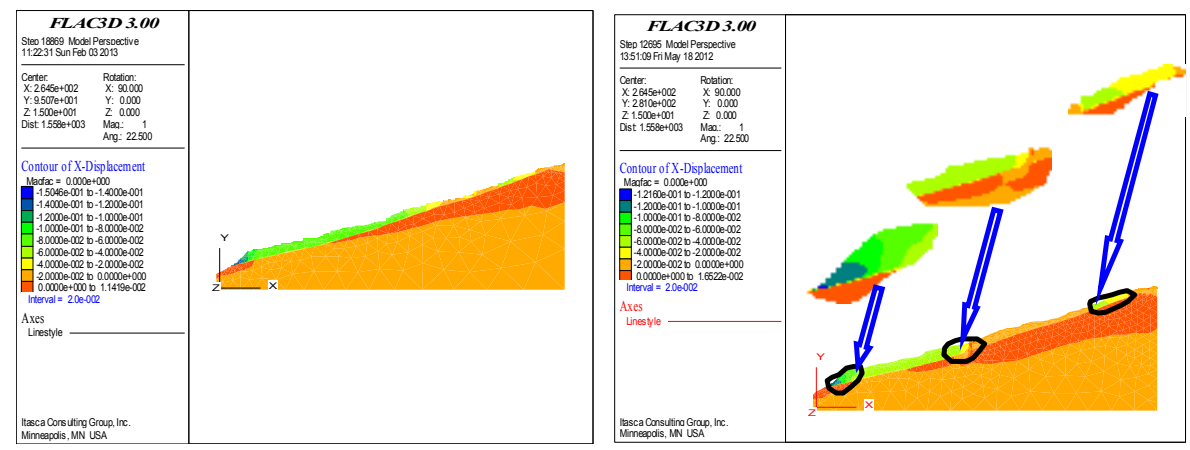

Fig.3 Contour map of displacement in nature

Fig.4 displacement after anti-slide pile constructing

The local of natural slope landslide's trailing edge have $10 \mathrm{~cm}$ displacement. The maximum of the displacement is $15 \mathrm{~cm}$ (fig 4) in the landslide body that located in the landslide leading edge. After the anti-slide pile construction, it can be seen from the contour map the displacement of the landslide trailing edge is less than $6 \mathrm{~cm}$ (fig 5), the displacement of the landslide gradually to increase that in front 
of anti-slide pile. The maximum displacement is $12 \mathrm{~cm}$ that located in the landslide leading edge, which accord with on-site field investigation in March 2011 (fig 6). The Landslide trailing edge 's crack about 5 to $7 \mathrm{~cm}$, the maximum of middle and lower's crack is $15 \mathrm{~cm}$, Illustrates the calculation result of the landslide model that after anti-slide pile construction is believable. After anti-slide pile construction, displacement nephogram mutagenesis oblique type of "L" shape cloud, anti-slide pile resistance slip effect is obvious. The displacement of landslide is smaller than the pile not construction, the Contour map of displacement mutagenesis as oblique type of "L" shape, that means the resistance slip effect of anti-slide pile is obvious.

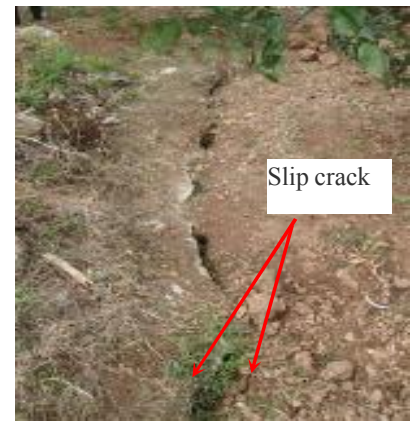

A The trailing edge 's crack

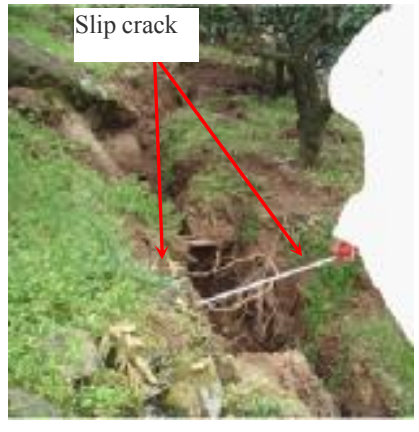

b The middle and lower's crack

Fig.5 The measurement of crack figure after anti-slide pile construction
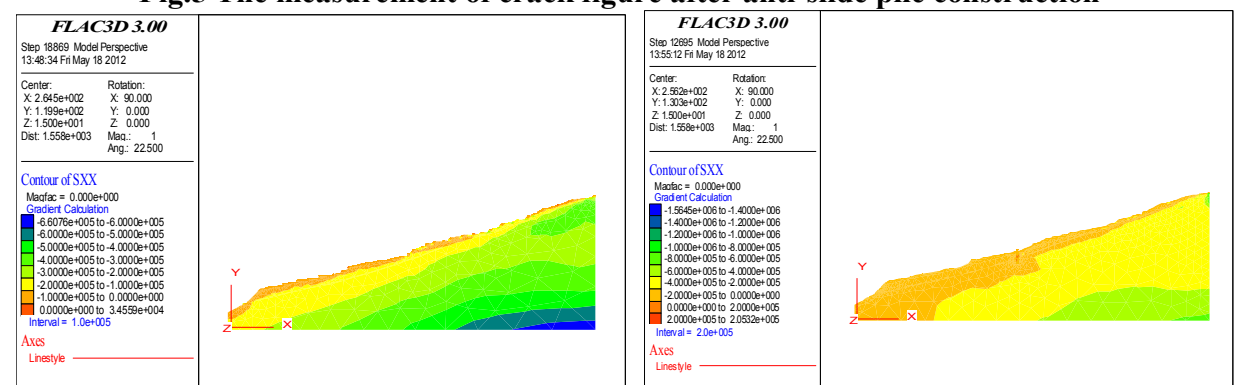

Fig.6 Contour map of shear stress in nature Fig.8 Contour map of shear stress after anti-slide pile construction

\section{Shear stress analysis}

From the shear stress nephogram (fig 7), the maximum shear stress of the slip band is $0.45 \mathrm{MPa}$, maximum shear stress of the landslide mass is $6.6 \mathrm{MPa}$ that located at the bottom of the ridge. After anti-slide pile construction, the maximum shear stress of slip band is less than $0.2 \mathrm{MPa}$, the shear stress increase around anti-slide pile. The Contour map of shear stress mutagenesis as oblique type of "L" shape too, that means the resistance slip effect of anti-slide pile is obvious. set up a series of monitoring points in the slip band position of that two models, the monitoring point set between $3 \#$ and $4 \#$ pile in order to facilitate analysis the changes of soil's displacement and stress between piles.
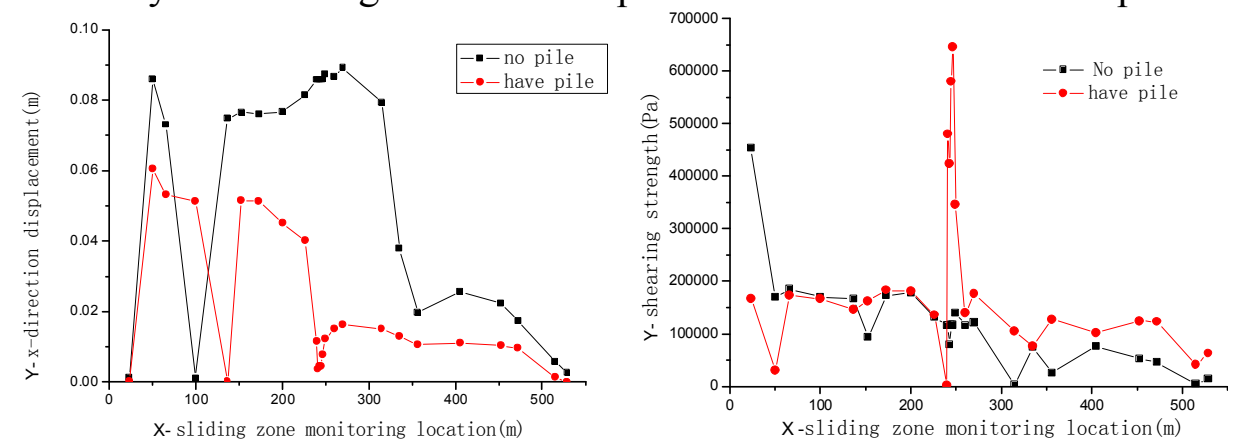

Fig.7 Horizontal displacement analysis of sliding zone 's monitoring stations

Fig.10 Shear stress analysis of sliding zone 's monitoring stations

\section{Monitoring result analysis}

Set up a series of monitoring points in the slip band position of that two models, the monitoring point set between $3 \#$ and $4 \#$ pile in order to facilitate analysis the changes of soil's displacement and stress that between piles. The anti-slide pile's position is between $240 \sim 243 \mathrm{~m}$ in the $\mathrm{x}$ direction. By the 
fig 9, anti-slide pile has obvious resistance slide effect, it's makes the $\mathrm{X}$ direction displacement decreases obviously, especially near the pile, from $90 \mathrm{~mm}$ that the pile pre-construction changed to under $10 \mathrm{~mm}$ when the pile after-construction. the shear stress value changed small in the landslide leading edge whatever the pile construction or not, after the pile construction the shear stress value of soil nearby the pile is increased 6 times than there without pile, in the Landslide trailing edge the shear stress value of the pile after-construction is higher that pre-construction, analysis that the pile play a important part in resistance slide effect, Lead to the soil that after pile resistance sliding, the pile and soil "coupling" resistance slip.

\section{Summary}

(1). Taking majiagou 1\# landslide as an example, establish numerical model, and considering the pile-soil contact surface properties, set up a series of monitoring point in sliding zone Analysis of the anti-slide pile resistance slide effect, compared to the wild field observation data demonstrates the reliability of numerical model.

(2). After the anti-slide pile construction, the maximum displacement of the landslide is smaller than before, the contour map of displacement and shear stress mutagenesis as oblique type of "L" shape, that means the resistance slip effect of anti-slide pile is obvious.

(3). Monitoring results shows, anti-slide pile has obvious resistance slide effect, make the $\mathrm{X}$ direction displacement decreases obviously, especially near the pile, the pile and soil "coupling" resistance slip.

(4). Can use the three-dimensional numerical analysis software FLAC3D to analysis the anti-slide pile resistance effect and coupling effect of soil-pile interaction, provides a feasible analysis method for the study of landslide prevention and control effect that has the same engineering geological conditions.

\section{References}

[1] YIN Yue-ping. The theory and practice of landslide controlling engineering in China[J].Hydrogeology and Engineering Geology, 1998, (1):5-9. (In Chinese)

[2] FENG Wen-juan,JU Xiao-dong.Design method for anti-slide piles based on FLAC3D[J].Journal of Geotechnical Engineering,2011,33(supp2): 256-259. (In Chinese)

[3] LI Shao-jun,CHEN Jing,LIAN Cao.Mechanical model of soil arch for interaction of piles and slope and problem of pile spacing[J].Rock and Soil Mechanics, 2010,31(5): 1352-1358. (In Chinese)

[4] TERZAGHI K. Theoretical soil mechanics[M]. New Youk: JohnWiley\&Sons, 1943.

[5] OSSCHER B, PETER J, GRAY, DONALD H. Soil arching in sandy slopes[J]. Journal of Geotechnical Engineering, 1986, 112(6): 626-645.

[6] LADANYI B, HOYAUX B. A study of the trap door problem in a granular mass[J]. Canadian Geotechnical Journal, 1969, 6(1): 1-14.

[7] WANG W L, YEN B C. Soil arching in slopes[J]. Journal of the Geotechnical Engineering Division, ASCE, 1974, 100 (GT1): 61-78.

[8] KOUTSABELOULIS N C, GRIFFITHS D V. Numerical modeling of the trap door problem[J]. Geotechnique, 1989, 39(1): 77-89.

[9] CHEN C Y, MARTIN G K. Soil-structure interaction for landslide stabilizing piles[J]. Computers and Geotechnics, 2002, (29): 363-386.

[10] Chen Yuming,Xu Dingping. FLAC/FLAC3D Foundation and engineering examples [M]. China Water Conservancy and Hydropower Press, 2008. (In Chinese) 